\title{
Competencias digitales en docentes de la educación pública: una revisión sistemática
}

\author{
Luque Ramos, Roxana del Carmen \\ roqueluqueramos2020@gmail.com
}

Mg. Jiménez Álvarez, Jyon Frankie ifjimenez19@hotmail.com

Perú

\section{RESUMEN}

El objetivo fue analizar el impacto de las competencias digitales en docentes de la educación básica. Para ello se ha precedido con la metodología establecida en la Declaración PRISMA, Para ello, se realizaron búsquedas combinadas mediante el uso de palabras claves en bases a la información científica de Scopus, EBSCO, Proquest, Springer y otros, en un periodo de tres años. Durante la investigación se han trabajado con flujo de información de las diferentes fases de la declaración Prisma estas son el proceso de identificación, cribado, elegibilidad e inclusión se desarrolló en cuatro meses. De los 149 estudios que arrojó la búsqueda especializada, solamente se incluyeron 15 . Criterios de inclusión: artículos de investigación, revisiones sistemáticas y estudios de caso, se orientó a la revisión y recopilación sistemática de las investigaciones que se realizaron sobre las competencias digitales en docentes de la educación básica. Criterios de exclusión: se excluyeron aquellas investigaciones que no fueron sometidas a un proceso de revisión, con deficiencias metodológicas o que no demostraron relación o interrelación entre las variables mencionadas anteriormente. Los resultados indican que las competencias digitales en docentes de la educación básica; han sido determinantes en los procesos de enseñanza y aprendizaje.

Palabras clave: competencia digital; docentes de la educación básica. 


\title{
Digital competences in public education teachers:
}

\section{A systematic review}

\begin{abstract}
The objective was to analyze the impact of digital competences in basic education teachers. To do this, the methodology established in the PRISMA Declaration has been preceded. To do this, combined searches were carried out using keywords based on scientific information from Scopus, EBSCO, Proquest, Springer and others, in a period of three years. . During the investigation, they have worked with the information flow of the different phases of the Prisma declaration, these are the identification, screening, eligibility and inclusion process that took place in four months. Of the 149 studies that the specialized search yielded, only 15 were included. Inclusion criteria: research articles, systematic reviews and case studies, it was oriented to the systematic review and compilation of research carried out on digital skills in teachers of basic education. Exclusion criteria: those investigations that were not subjected to a review process, with methodological deficiencies or that did not demonstrate a relationship or interrelation between the variables mentioned above were excluded. The results indicate that digital skills in basic education teachers; They have been decisive in the teaching and learning processes.
\end{abstract}

Keywords: digital competence; teachers of basic education.

Artículo recibido: 10 Setiembre. 2021 Aceptado para publicación: 15 Octubre. 2021 Correspondencia: roqueluqueramos2020@ gmail.com

Conflictos de Interés: Ninguna que declarar 


\section{INTRODUCCION}

Es de conocimiento para todo profesional que hoy en día vivimos e interactuamos en una aldea global, que esta interconectada por las tecnologías de la información y la comunicación, ya que toda información y suceso se hace de conocimiento público en tiempo real, gracias al desarrollo que han alcanzado estas tecnologías comunicativas. Por ello, en las universidades y centros de formación profesional no existe un egresado que no haya llevado un curso de actualización o formación básica en TIC, porque constituye un requisito primordial para conseguir posicionarse laboralmente y tener presencia en una sociedad digital. Las universidades deben formar a los futuros docentes, siendo necesario que se les infunda desde sus años de formación inicial, la necesidad de formarse en competencias digitales. (M. Ruiz, 2020)

$\mathrm{Y}$ es que se sabe, que cuando hablamos de competencias digitales no solo nos referimos al uso de la computadora para realizar un documento en Word o Excel que existen múltiples usos que darse en la labor directiva pedagógica, funciones que apunten al uso de las TIC para rediseñar o producir contenidos o datos para atender la realidad que presenta cada institución educativa. (Rodríguez, 2014)

Por ejemplo, crear comunidades virtuales para trabajar colaborativamente en la construcción de documentos de gestión de manera sincrónica o asincrónica, realizar una encuesta online para recoger aportes de los padres de familia o sensaciones de satisfacción con el servicio prestado, solicitud o entrega virtual de documentos a padres de familia, uso de EDpuzzle para la edición de videos propios o de la red en talleres con docente, padres de familia o estudiantes ajustándolos a sus necesidades, publicaciones en blogs interconectados a comunidades docentes de aprendizaje, asignación de tareas para diversos grupos de la comunidad educativa, etc.

Es así que, dentro de las actividades se enseñanza aprendizaje, en el presente año, se ha evidenciado que muchos docentes no tienen desarrolladas sus habilidades en el uso de las TIC, no pueden interactuar con la información y no tienen frecuencia en el uso de las diferentes herramientas tecnológicas. Si estos docentes pudieran mejorar sus habilidades en el uso de las TIC, entonces la comunicación será más rápida y el intercambio de información sería más viable, pudiendo atenderse más rápido cualquier problemática que surgiera dentro de las instituciones educativas. Dominar las TIC implica empoderase, ser un individuo capaz, autónomo, preparado, eficiente, comprometido responsable, crítico y 
reflexiva ya que puede escoger y cambiar la información que desee, utilizando las distintas herramientas tecnológicas. La noción de competencia aparece junto con la gestión contemporánea de los recursos humanos, cuando estudios empíricos realizados en EE. UU durante los años sesenta ya se exigía cierto dominio tecnológico en el uso de las TIC.

Existen cinco áreas que atiende la competencia digital: la información y alfabetización informacional que le permite interactuar con la información que circula en la redes, segundo la comunicación y colaboración de la comunidad en entornos digitales, tercero la creación de contenido digital donde pueden crear imágenes, videos y archivos de multimedia, cuarto la seguridad que está relacionada con la protección de la información y quinto la resolución de problemas que permite identificar problemas y resolverlos haciendo uso de las herramientas digitales. (Romero et al., 2020)

En respuesta a la nueva sociedad del conocimiento e información, así como a las exigencias del trabajo remoto actual, los docentes tienen el compromiso de desarrollar las competencias digitales, este propósito les permitirá el logro de habilidades en la utilización de las herramientas TICs, además de la adquisición de una actitud crítica reflexiva en la creación y uso de los contenidos, utilización ética y legal. De esta manera los docentes de la educación básica deben comprometerse a utilizar estas herramientas, debiendo ser creativos en la aplicación de estos recursos digitales y los medios de comunicación en las diferentes áreas, con sus respectivos campos temáticos. La pregunta de investigación es la siguiente: "Cual es el nuevo rol del docente en la nueva sociedad del conocimiento e información, así como que factores influyen en los cambios en la práctica de los docentes en la utilización de las TICs". Asimismo, el presente artículo tiene como objetivo: "Analizar el impacto de las competencias digitales en docentes de la educación básica.”.

\section{METODOLOGÍA}

La revisión se desarrolló según la lista de verificación de 27 elementos y el diagrama de flujo previsto en la Declaración PRISMA. El levantamiento de información se realizó en Scopus, EBSCO, Proquest, Springer y otros, el periodo meta fue desde 01/12/2017 hasta 01/12/2020, el periodo de identificación, cribado, elegibilidad e inclusión se desarrolló en tres meses. Las palabras principales de búsqueda fueron "digital competences" y “teachers basic education”. La búsqueda arrojó un total de 149 documentos que fueron 
procesados según la metodología mencionada, siendo seleccionados 15 investigaciones del total. Criterios de inclusión: artículos de investigación, revisiones sistemáticas y estudios de caso, se orientó a la revisión y recopilación sistemática de las investigaciones que se realizaron sobre las competencias digitales en docentes de la educación básica. Criterios de exclusión: se excluyeron aquellas investigaciones que no fueron sometidas a un proceso de revisión, con deficiencias metodológicas o que no demostraron relación o interrelación entre las variables mencionadas anteriormente.

\section{Este es mi diagrama prisma}

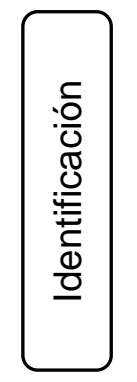

\section{Registros identificados mediante} la búsqueda en Scopus $(n=82)$

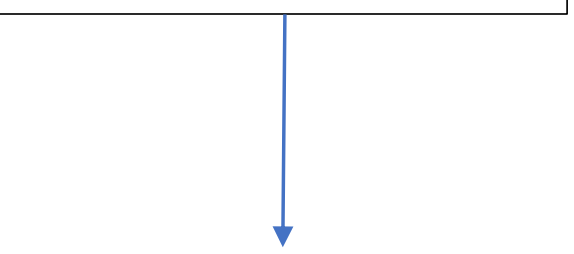

Registros después de eliminar duplicados $(n=124)$
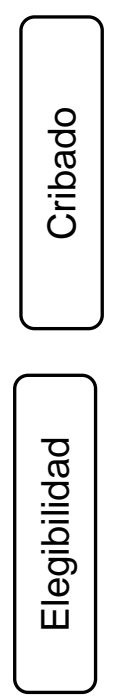

$\begin{array}{r}0 \\ \text { 음 } \\ \frac{3}{3} \\ \text { ㅌ } \\ \hline\end{array}$
Registros adicionales identificados en otras bases de datos $(n=67)$
Artículos excluidos a nivel de título y resumen $n=82$

Artículos a texto completo excluidos por incumplir los Criterios de inclusión declarados en la metodología $(n=27)$

Figura 1. Diagrama de flujo de selección de unidad de análisis PRISMA

( Moher et al., 2014) 
Tabla 1. Investigaciones incluidas en la revisión sistemática

\begin{tabular}{|c|c|c|c|c|c|c|c|c|}
\hline \multirow[b]{2}{*}{ No } & \multirow{2}{*}{$\begin{array}{l}\text { Autores/ Año/ } \\
\text { Título/ Publica }\end{array}$} & \multicolumn{5}{|c|}{$\begin{array}{l}\text { COMPETENCIAS DIGITALES EN DOCENTES DE LA } \\
\text { EDUCACION PUBLICA }\end{array}$} & \multicolumn{2}{|c|}{ Metodología } \\
\hline & & $\begin{array}{l}\text { Información y } \\
\text { alfabetización } \\
\text { informacional }\end{array}$ & $\begin{array}{l}\text { Comunicación } \\
\text { y colaboración }\end{array}$ & $\begin{array}{l}\text { Creación de } \\
\text { contenidos }\end{array}$ & $\begin{array}{l}\text { Seguir- } \\
\text { dad }\end{array}$ & $\begin{array}{l}\text { Resolución de } \\
\text { problemas }\end{array}$ & $\begin{array}{l}\text { Tipo de } \\
\text { estudio }\end{array}$ & $\begin{array}{l}\text { Instrumento, } \\
\text { técnica o } \\
\text { método } \\
\end{array}$ \\
\hline 01 & $\begin{array}{l}\text { (Pérez et al., } \\
2020)\end{array}$ & $X$ & $X$ & & & & Cuantitativo & Encuesta \\
\hline 02 & $\begin{array}{l}\text { (Sánchez et al., } \\
\text { 2020) }\end{array}$ & $X$ & $X$ & & & $X$ & Cuantitativo & Encuesta \\
\hline 03 & $\begin{array}{l}\text { (Ruiz et al., } \\
2020 \text { ) }\end{array}$ & $X$ & $X$ & $\mathrm{X}$ & & & Mixta & $\begin{array}{l}\text { Cuestiona- } \\
\text { rio }\end{array}$ \\
\hline 04 & $\begin{array}{l}\text { Martínez y } \\
\text { Garcés, 2020) } \\
\end{array}$ & $X$ & $X$ & $X$ & $X$ & $X$ & Cuantitativo & Encuesta \\
\hline 05 & $\begin{array}{l}\text { (Romero et al., } \\
2020 \text { ) }\end{array}$ & $X$ & $X$ & $\mathrm{X}$ & $X$ & $X$ & Cuantitativo & Encuesta \\
\hline 06 & $\begin{array}{l}\text { (Díaz \& } \\
\text { Berrocoso, } \\
2020) \\
\end{array}$ & & $X$ & & & & Cualitativa & Entrevista \\
\hline 07 & $\begin{array}{l}\text { Ruiz, } \quad \text { M. } \\
(2020) .\end{array}$ & $X$ & $X$ & $X$ & $\mathrm{X}$ & $X$ & Cuantitativo & Encuesta \\
\hline 08 & $\begin{array}{l}\text { (López et al., } \\
\text { 2019) }\end{array}$ & $X$ & $X$ & $X$ & & $X$ & Cuantitativo & Encuesta \\
\hline 09 & $\begin{array}{l}\text { (Villarreal et } \\
\text { al., 2019) }\end{array}$ & $\mathrm{X}$ & $X$ & $\mathrm{X}$ & & & Mixta & Cuestionario \\
\hline 10 & $\begin{array}{l}\text { (Flores y Roig, } \\
\text { 2019) }\end{array}$ & $X$ & $X$ & $X$ & & & Cuantitativo & Encuesta \\
\hline 11 & $\begin{array}{l}\text { (Fuentes et al., } \\
\text { 2019) }\end{array}$ & $X$ & $X$ & $X$ & $X$ & $X$ & Cuantitativo & Encuesta \\
\hline 12 & $\begin{array}{l}\text { Bustos \& } \\
\text { Gómez, 2018) }\end{array}$ & & $X$ & $\mathrm{X}$ & & & Cuantitativo & Encuesta \\
\hline 13 & (Román, 2018) & $X$ & $X$ & $\mathrm{X}$ & & & Cuantitativo & Encuesta \\
\hline 14 & $\begin{array}{l}\text { (Fernández et } \\
\text { al., 2018) }\end{array}$ & $X$ & $X$ & $X$ & & & Cuantitativo & Encuesta \\
\hline 15 & (Boudet, 2017) & $X$ & $X$ & $X$ & & & Cuantitativo & Encuesta \\
\hline
\end{tabular}

La Tabla 1 muestra la distribución de los resultados generales de la búsqueda.

El 86,6 \% de los estudios responden a la subcategoría Información y alfabetización informacional (13 de los estudios demuestran interrelación con las demás subcategorías). El 100,00\% de los estudios responden a la subcategoría comunicación y colaboración (15 de los estudios se interrelación con las demás subcategorías). El 80,00 \% de los estudios responden a la subcategoría creación de contenidos digitales (12 de los estudios se interrelación con las demás subcategorías). El 26,6 \% de los estudios responden a la subcategoría seguridad (4 de los estudios se interrelación con las demás subcategorías). El $40.00 \%$ de los estudios responden a la subcategoría resolución de problemas (6 de los estudios se interrelaciona con las demás categorías). El 13,30\% de los estudios incluidos 
fueron de enfoque cualitativo, un $6,7 \%$ de enfoque mixto y finalmente el $80,00 \%$, cuantitativo.

$\mathrm{Al}$ analizar los resultados, se encontró que información y alfabetización informacional, comunicación y colaboración, creación de contenidos, seguridad y resolución de problemas, fueron subcategorías centrales determinantes para el estudio de las competencias digitales en docentes de la educación pública. Las mismas, con el propósito de desarrollar una discusión focalizada y coherente con el objetivo de esta investigación, se discuten a continuación.

\section{DISCUSIÓN}

En relación con la subcategoría de información y alfabetización informacional se producen evidencias mejoras en estas habilidades para la búsqueda, análisis e identificación y selección de información digital más importantes que requiere, a sus estudiantes solicitan que indaguen y diferencien los tipos de información (Baulet,2017). Los docentes debemos desarrollar la habilidad de buscar, organizar y analizar la información. Debemos localizar, también almacenar los datos y contenidos digitales, e identificar las posibilidades de incorporar a nuestras clases diarias, es decir que los docentes seamos capaces de realizar la búsqueda de información y selección en la red, seleccionando los recursos más efectivos en el logro de los aprendizajes de nuestros estudiantes.

Martínez (2020), encontró que los docentes, tienen habilidad para localizar información digital en la red que sea útiles para sus clases, identificando los nuevos conocimientos, clasificándolos adecuadamente para su revisión posterior, bien sea desde un almacenamiento local o desde la misma web. Los docentes dominan la herramienta digital para la búsqueda de información debido a que poseen la capacidad para seleccionar los recursos digitales de acuerdo a las necesidades de aprendizaje. A nuestros estudiantes debemos trasladar esta habilidad del almacenamiento y recuperación de información, datos y contenidos digitales. Los docentes debemos ser competentes para el almacenamiento de archivos y utilización de diversos métodos para compartir información con sus pares y estudiantes.

En cuanto a la subcategoría de comunicación y colaboración, Bustos \& Gómez, (2018), encuentran que una de las problemáticas manifestadas en forma reiterada por los docentes es la comunicación con sus colegas y sus estudiantes, esto se originado por una carencia 
de integración entre las herramientas tecnológicas con las estrategias pedagógicas, y de las redes en su trabajo cotidiano. Los docentes debemos estar capacitados para compartir recursos a través del internet, así como interactuar en comunidades virtuales. Asimismo, debemos utilizar una variedad de recursos y/o aplicaciones que deben incorporan en nuestra planificación de las sesiones diarias de clase, de manera definitiva para la mejora de los aprendizajes, los docentes debemos ser proactivo en la difusión de nuestros recursos educativos, fortaleciendo esta habilidad e incentivando la utilización de las TICS entre nuestros estudiante y equipo docente de nuestras instituciones educativas. Transformándoos en seres activos, dinámicos y participando en diferentes medios virtuales y siendo consciente de los potenciales de la tecnología en la mejora de los aprendizajes.

Por su parte, Ruiz et al. (2020), señala que es fundamental el trabajo colaborativo y de acompañamiento en el aprendizaje profesional de los docentes en sus competencias en la utilización de las tecnologías. Debemos debe compartir espacios digitales de trabajo con otros compañeros de trabajo, compartiendo estrategias, creando documentos en línea, participando en videoconferencias para fortalecer la practica pedagógica, debiendo ser capaz de identificar las conductas inadecuadas e intervenir en casos de bulling virtual o ciberacoso.

En la subcategoría de creación de contenidos, para Ruiz (2020), es importante que los docentes adquieran estas habilidades porque ello les va a permitir la adaptación de los recursos y los contenidos a las características de sus estudiantes, por lo cual se requiere, que el docente deba crear contenidos en diferentes formatos y espacios, así como la creación de proyectos y materiales didácticos digitales propias con la participación activa de sus estudiantes. Así también la presentación de trabajos en formatos digitales, creación de blogs del centro, uso de aplicaciones para el aprendizaje de diferentes campos de estudio, así como el manejo de la Internet con propósitos educativos.

Villareal, et al. (2020), concluyen su estudio señalando, la necesidad de generar en los docentes compromisos en la actualización de sus conocimientos e información, así mismo, promover la producción de contenidos digitales que desarrollen nuevas competencias en los estudiantes. Los docentes estamos comprometidos efectivamente con las TICs las cuales son herramientas eficaces que puede ser utilizada para apoyar el proceso de enseñanza aprendizaje, pudiendo aplicarse en propuestas educaciones tanto 
presenciales como virtuales. La creación de contenidos y materiales con soporte de nuevas tecnologías está atada al nuevo rol de los docentes y de los estudiantes, que permitan a los docentes transmitir conocimientos en un contexto educativo dominado por la cultura audiovisual y que, a su vez, el estudiante construya sus propios aprendizajes, gestionando su aprendizaje de manera autónoma. Así mismo, el aprendizaje sobre las diferentes licencias sobre la utilización de los datos, respetando tanto los derechos de autores de los materiales utilizados, así como del contenido propio.

En cuando a la subcategoría seguridad de la competencia digital, a modo general, se encuentra que los docentes realizan acciones de manera regular acciones que implican protección personal, de datos y de identidad digital. Ruiz (2020), Los docentes deben proteger la información (fundamentalmente la personal), adoptando medidas de seguridad y la utilización con responsabilidad de la tecnología. De esta manera, los docentes deben establecer contraseñas seguras, además de instalar antivirus avanzado en el que protege los dispositivos para la identificación de fallos. De la misma forma se debe proteger la privacidad de cada persona incidiendo en su importancia entre los estudiantes. Por último, abordar la subcategoría de la seguridad digital es muy amplio; no sólo actúan factores instrumentales que indiquen buenas prácticas de utilización de las TICs, sino también psicológicos y sociales.

Finalmente, se han obtenido en la Subcategoría de resolución de problemas. Romero (2020), concluye que los docentes deben reforzar esta subcategoría que está relacionada con el aprendizaje de resolución de problemas a través de medios digitales, utilizando la tecnología de forma creativa, para generar conocimientos, Al respecto, los docentes debemos incluir en la resolución de problemas los problemas técnicos de los diferentes dispositivos y herramientas, también el docente ser capaces de elegir la herramienta que más se conveniente a nuevas necesidades que van surgiendo a lo largo de nuestros trabajo pedagógico.. Otro aspecto es la innovación y uso de la tecnología digital de forma creativa por ello es imprescindible la actualización de conocimientos y el contacto habitual con iniciativas innovadoras. Definitivamente la formación en competencias digitales es algo fundamental para los docentes actuales tanto para la mejora de su forma de enseñar como para conectarse con sus estudiantes.

Es una subcategoría de resolución de problema es muy ambiciosa, se enfoca, fundamentalmente, en la identificación de necesidades y recursos digitales, la toma de 
decisiones en el momento de utilizar la herramienta digital adecuada, de acuerdo con la necesidad o finalidad, resolviendo problemas conceptuales con medios digitales, resolviendo problemas técnicos, utilizando creativamente la tecnología, actualizando las competencias propias y la de demás.

Considerando la coyuntura actual se hace necesario dinamizar las competencias digitales que permitan alcanzar los logros académicos en los niveles de la educación.

Ruiz (2020) encuentra en su trabajo que existen desigualdades en el acceso a las TICs entre lo rural y lo urbano, lo que connleva al incumoplimiento de los principios establecidos por el Marco Comun Europeo, en este sentido, difiere con Romero et al., 2020), quienes en sus resultados de su investigación encuentran mejoras en las cinco áreas de la competencia digital especificadas por el Marco Común de las Competencias Digitales Docentes, con un gran tamaño de efecto. Concluyen que el experimento educativo implementado ha permitido incrementar el nivel de competencia digital de los futuros docentes. Los docentes debemos ser capaces de integrar los contenidos del CNEB con el apoyado de la tecnología, esto implica una adecuada manejo de las tecnologías en las aulas que incidan en mejoras del proceso enseñanza - aprendizaje y, en definitiva, el docente debe ser capaz de utilizar e integrar las TIC adecuadamente en las actividades de enseñanza-aprendizaje.

Ruiz et al., (2020), en su estudio encuentran, que el interés de los docentes por comprender e integrar las competencias digitales. Los docentes tenemos que fortalecer nuestras las competencia digital para facilitar el aprendizaje y mejorar la enseñanza en nuestras clases, para lograr que nuestros estudiantes puedan dinamizar y compartir experiencias en línea con otros estudiantes de diversas latitudes.

\section{CONCLUSIONES}

El desarrollo de las Tecnologías de la Información y la Comunicación (TICs) ha generado grandes cambios en la sociedad actual, un sector tocado es el educativo. Los procesos educativos han debido asimilar la utilización de las herramientas tecnológicas, para su aplicación en la educación actual. La utilización de estas herramientas tecnológicas supone que los docentes deben adquirir competencias digitales, este reto, en las condiciones actuales es perentorio.

El impacto de las competencias digitales en los docentes de la educación básica, supuso la necesidad de promoción de manera acelerada del desarrollo de las competencias 
digitales en los docentes; se requirió, además, que los docentes cuenten las herramientas necesarias que les permitan integrar estas al proceso de enseñanza aprendizaje; de igual modo la capacitación para el uso responsable y ético de estas herramientas.

Finalmente, se hace necesario que el órgano normativo del sector educación, implemente políticas formativas de TICs y planes de estudio que incorporen contenidos para el desarrollo de las competencias digitales en los centros de educación superior que forman a los futuros docentes.

\section{REFERENCIAS BIBLIOGRAFICAS}

Boudet, J. (2017). Evaluación de la competencia digital docente en la comunidad autónoma de Aragón. Revista Electronica de Investigacion Educativa, 19(4), 73-83.

Bustos, H., \& Gómez, M. . (2018). La competencia digital en docentes de preparatoria como medio para la innovación educativa. CPU-e, Revista de Investigación Educativa, 26, 6686.

Díaz, M. ., \& Berrocoso, J. . (2020). Perfiles docentes en un contexto de transformación digital en la escuela. Bordon. Revista de Pedagogia, 72(1), 151-173.

Fernández, E., Leiva, J., \& López, E. (2018). Competencias digitales en docentes de Educación Superior. Revista Digital de Investigación En Docencia Universitaria, 12(1), 213-231.

Flores, C., \& Roig, R. (2019). Performance y formación educativa en competencias digitales en clases no presenciales durante la pandemia covid-19. In Revista Iberoamericana de Educacion Superior (Vol. 10, Issue 27, pp. 151-171). Universidad Nacional Autonoma de Mexico.

Fuentes, A., López, J., \& Pozo, S. (2019). Análisis de la Competencia Digital Docente: Factor Clave en el Desempeño de Pedagogías Activas con Realidad Aumentada. REICE: Revista Iberoamericana Sobre Calidad, Eficacia y Cambio En Educación, ISSN-e 1696-4713, Vol. 17, No. 2, 2019, Págs. 27-42, 17(2), 27-42.

Martínez, J., \& Garcés, J. (2020). Competencias digitales docentes y el reto de la educación virtual derivado de la covid-19. Educación y Humanismo, 22(39), 1-16.

Moher, D., Liberati, A., Tetzlaff, J., \& Altman, D. G., \& P. G. (2014). Ítems de referencia para publicar revisiones sistemáticas y metaanálisis: la Declaración PRISMA. In Rev Esp Nutr Hum Diet (Vol. 18, Issue 3). http://medicine.

Pérez, A., Iglesias, A., \& Berrocal, V. (2020). Competencia digital docente para la reducción de la brecha digital: Estudio comparativo de España y Costa Rica. Trípodos., 46, 77-96.

Román, Y. (2018). Entornos virtuales de aprendizaje y desarrollo de competencias digitales en los docentes. In Rev Yachay (Vol. 7, Issue 7).

Romero, C., Buzón, O., \& de Paz, P. (2020). Mejorar la competencia digital de los futuros 
profesores mediante metodologías activas. Sustainability (Switzerland), 12(18).

Ruiz, A., Medina, M., Perez, E., \& Medina, A. (2020). La formación del profesorado universitario: la competencia digital. Pixel-Bit, Revista de Medios y Educacion, 58, 181215.

Sánchez, S., Belmonte, J., Moreno, A., \& Hinojo, F. . (2020). Aprendizaje invertido y competencia digital: una conexión docente necesaria para su desarrollo en la educación actual. Revista Electronica Interuniversitaria de Formacion Del Profesorado, 23(2), $127-141$.

Villarreal, S., García, J., Hernández, H., \& Steffens, E. (2019). Competencias y transformaciones docentes en educación en la era digital. Formacion Universitaria, 12(6), 3-14. 